\title{
North-South asymmetry of equatorial ionospheric anomaly computed from the IRI model
}

\author{
Sanjay Kumar \\ Banaras Hindu University, Varanasi - 221005, India \\ Article history: received July 31, 2019; accepted December 18, 2019
}

\begin{abstract}
Using the electron density from the IRI-2016 model and processed results of the in-situ measurements (GRACE and CHAMP) in Xiong et al. (2013), the morphological features of the equatorial ionization anomaly (EIA) such as its magnitude and inter-hemispheric asymmetry have been studied during 2001-2009. The effect of solar activity on ability of the IRI-2016 model to predict the EIA parameters is studied and the results are compared with in-situ measurements from GRACE and CHAMP during high and low solar activity phases. The IRI-2016 generally follows the same latitudinal asymmetry (ACL - Asymmetry of Crest Latitude) at $400 \mathrm{~km}$ (the same trend of ACL as for CHAMP) while it tends to be asymmetric towards the North at $480 \mathrm{~km}$ (GRACE) (Positive ACL). In addition, during June solstice the IRI-2016 model shows larger departure from observation after 19:00 UT with a larger difference during high solar activity than that during the low solar activity. Also the IRI-2016 model failed to predict significantly North-South asymmetry (southern crest disappears) in the electron density for high solar activity period. This is probably the reason for larger discrepancy of observations and the IRI-2016 model particularly during the summer solstice of the high solar activity period. This suggests that data input in the IRI model particularly in the equatorial- and low-latitude regions are required so that it could better predict the location of EIA crests.
\end{abstract}

Keywords: EIA, Solar activity, The IRI-2016 model.

\section{Introduction}

The equatorial ionospheric anomaly (EIA) is characterized, in terms of latitudinal distribution of ionization, by a trough at the magnetic equator and crests at about $\pm 17^{\circ}$ magnetic latitude and crest to trough ratio of about 1.6 in daytime peak electron density [Appleton, 1946, Balan et al., 2018]. The position of the crests and crest-to-trough ratio vary with various geophysical conditions. Many theories, like the diffusion theory [Mitra, 1946; Rishbeth et al., 1963] and the electrodynamic drift theory [Martyn, 1955; Moffett and Hanson, 1965] have been known to explain the anomaly crest. Although Mitra [1946] suggested the role of diffusion in the development of EIA, the correct explanation was given after Martyn's electromagnetic drift theory which is based on upward plasma drift followed by diffusion. The fountain effect and associated anomaly can cover more than $30^{\circ}$ latitudes on either sides of the magnetic equator [Mannucci et al., 2005; Balan et al., 2011, 2018; Kumar et al., 2014a]. The perseverance of EIA into the nighttime hours depending on the season and solar activity is known to be produced by the post-sunset 


\section{Sanjay Kumar}

enhancement in the eastward electric field produced by the F-region dynamo action. This dynamo action, in turn, results from the eastward component of the F-region thermospheric wind blowing, with meanwhile the decreasing dawn-to-dusk E-layer Pedersen conductivity distribution [Heelis, 2004]. Balan and Bailey [1995] studied the plasma fountain including also neutral wind and they showed that the plasma velocity turns more pole-ward in that hemisphere, where the wind is pole-ward. The role of EIA in the initiation of ESF (Equatorial Spread-F) was discussed by Raghavarao et al. [1988] and they showed that the large crest to trough ratio of EIA in the 270-300 km altitude during the post-sunset 17:00 - 19:00 LT is favorable condition for occurrence of post sunset ESF. EIA is responsible for the global maximum values of the total electron content (TEC) over tropical latitudes which affect the radio propagation range determinations based on GPS satellite signals. It also contributes to the enhanced ionospheric scintillations effects produced by spread-F/plasma bubbles (depleted electron density region) irregularities on transionospheric radio wave (i.e. GPS signal) propagations [Abdu, 2005].

The formation of EIA can be seen in the electron density or TEC of the ionosphere and can exhibit diurnal, day-to-day, monthly, seasonal, semiannual, annual, and 11 year solar cycle variations [Huang et al., 1989; Rastogi and Klobuchar, 1990; Kumar and Singh, 2009; Kumar et al., 2012, 2014a]. Apart from these features the formation of the EIA in two hemispheres shows an interesting feature called inter-hemispheric asymmetry of two crests. Using the data from ground based ionosonde measurements over $5^{\circ} \mathrm{S}$ to $30^{\circ} \mathrm{N}$ along the Tamanrasset, Vila [1971] reported that the asymmetry in the EIA crests varied in latitude and local time and showed day-to-day variability. The EIA crest in the summer hemisphere was always found to exhibit larger changes. Using the hourly median latitudinal profile of electron content data over South East Asia, Walker [1981] showed that winter crest is dominant in the morning sector and decays more rapidly in the late afternoon sector. He further showed that the EIA crest asymmetries were found to be less significant during the solar maximum as compared to solar minimum. Using data from COSMIC (Constellation Observing System for Meteorology, Ionosphere and Climate) measurements, during July-August, 2006, Lin et al. [2007] exposed that apart from the asymmetry in neutral composition impacts, interaction between the summer to winter (also known as trans equatorial wind) neutral wind and strength of equatorial plasma fountain effect also play a significant role in producing the asymmetric evolution of EIA. Recently using the CHAMP and GRACE observations from 2001 to 2009, Xiong et al. [2013] have shown the seasonal and local time variation of the EIA magnitude as well as its inter-hemispheric asymmetry. They have also compared their results with the SAMI2 model.

In this paper our aim is to study the seasonal and annual variations of EIA crest parameters as defined by Xiong et al. [2013], inter-hemispheric asymmetries properties using the electron density of ionosphere computed from the IRI-2016 model and to compare the results with those reported by Xiong et al. [2013] using CHAMP and GRACE observations. Section 2 introduces the EIA parameters and data sets. Section 3 describes seasonal and local time variations of the EIA parameters and its comparison with CHAMP and GRACE observations. Finally, section 4 summarizes the results.

\section{Data, Model and Method}

The CHAMP satellite which stands for Challenging Minisatellite Payload, is a German satellite which was launched on 15 July 2000. The initial altitude of satellite was $\sim 456 \mathrm{~km}$ but by the end of 2009 its orbit decayed to $310 \mathrm{~km}$. The Planar Longmuir Probe (PLP) on-board satellites has been used to measure electron density as well as temperature at every $15 \mathrm{~s}$ [Reigber et al., 2000]. Gravity Recovery and Climate Experiment (GRACE) satellites comprising two spacecraft GRACE-A and GRACE-B. It was launched on 17 March 2002 into a near circular polar orbit. Initial altitude of this spacecraft was fixed of about $490 \mathrm{~km}$. Unlike the CHAMP the altitude of these satellites is quite stable and over the years and has remained stable around $480 \mathrm{~km}$. The two spacecraft follow each other by a distance of $\sim 170-220 \mathrm{~km}$ and the TEC between the two spacecraft can be obtained using the K-band ranging (KBR) data. On dividing the horizontal TEC by the distance between spacecraft the average electron density can be computed [Xiong et al. 2010].

The IRI model provides a series of ionospheric parameters e.g., electron density, electron temperature, ion composition, ion temperature and total electron content for any given latitude and longitude, time and date at altitudes ranging from 60 to $2000 \mathrm{~km}$. For F-peak model, the CCIR option is recommended for continental areas, whereas the URSI option is recommended over the ocean areas [Rush et al., 1989]. In this study, we have taken 


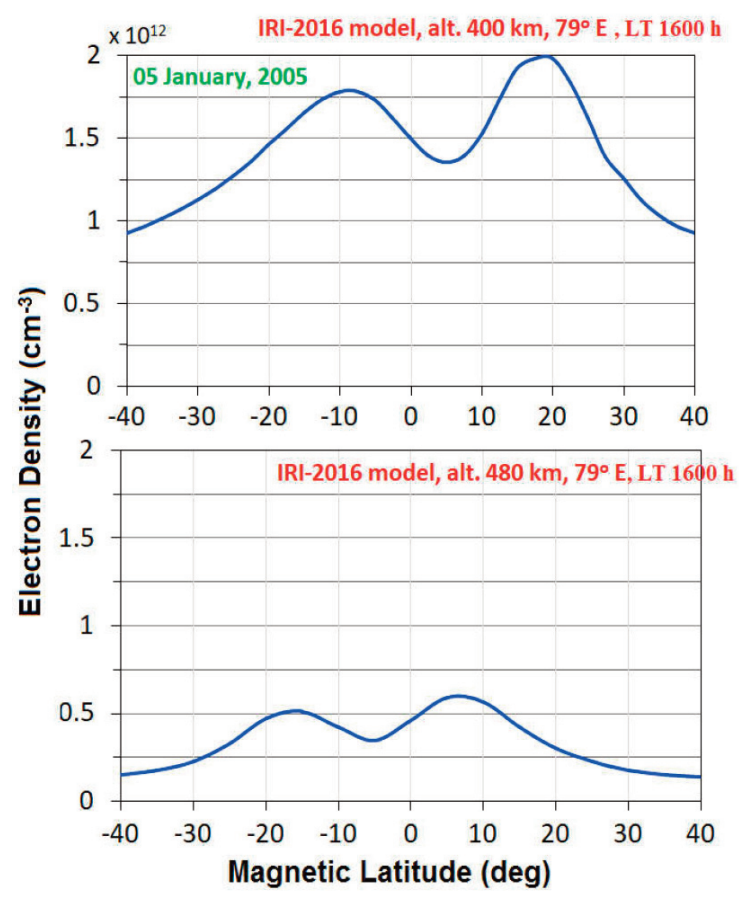

Figure 1. Latitudinal distribution of electron density estimated from the IRI-2016 model at 400 \& $480 \mathrm{~km}$ altitude, and $79^{\circ} \mathrm{E}$, showing north-south asymmetry in EIA.

latitudinal profiles of electron density data computed from the IRI-2016 model using CCIR coefficients at fixed altitudes of 400 and $480 \mathrm{~km}$ only. The electron density data is computed using the IRI-NeQ options for the topside $\mathrm{Ne}$ (Ne stands for electron density). Figure 1 shows a typical example of latitudinal profiles of electron density showing North-South asymmetry estimated from the IRI-2016 model. In the present study, the latitudinal profiles of electron density calculated from the IRI-2016 model in the range 10:00-22:00 LT have been used to estimate the EIA parameters represented by following equations [Xiong et al., 2013].

Mean Crest Intensity (MCI) is given by

$$
\mathrm{MCI}=\frac{\mathrm{Ne}_{\text {north_c }}+\mathrm{Ne}_{\text {South_c }}}{2}
$$

Crest-to-Trough Ratio (CTR)

$$
\mathrm{CTR}=\frac{\mathrm{Ne}_{\text {north_c }_{\text {c }}}+\mathrm{Ne}_{\text {South_c }}}{2 . \mathrm{Ne}_{\text {trough }}}
$$

Mean Crest Latitude (MCL)

$$
\mathrm{MCL}=\frac{\beta_{\text {north_c }}-\beta_{\text {south_c }}}{2}
$$

where $\mathrm{Ne}_{\text {north_c }}$ and $\mathrm{Ne}_{\text {south_c }}$ are electron densities of EIA crest in the two hemispheres and $\beta_{\text {north_c }}$ and $-\beta_{\text {south_c }}$ are the magnetic latitude of EIA crest in the two hemispheres.

Similar to the EIA magnitudes parameters the Asymmetry of the Crest-Intensity (ACI) as well as Asymmetry of Crest Latitude (ACL) are given by

$$
\mathrm{ACI}=\frac{\mathrm{Ne}_{\text {north_c }}-\mathrm{Ne}_{\text {south_c }}}{\mathrm{MCI}}
$$




$$
\mathrm{ACL}=\beta_{\text {north_c }}+\beta_{\text {south_c }}
$$

In this study all the above parameters have been computed using the IRI-2016 model. The data of the EIA parameters from GRACE and CHAMP observations has been taken from the Xiong et al. [2013] by digitizing the graph and compared with those estimated from the IRI-2016 model. First of all the crests and troughs have been identified from the latitudinal distribution of electron density data from the IRI-2016 model within latitudinal band of $\pm 30^{\circ}$ from the magnetic equator, at altitude of $400 \mathrm{~km}$ for CHAMP and $480 \mathrm{~km}$ for GRACE, then the derived EIA parameters were stored in different bins. To find out the crests and trough of the EIA, an automated detection of two maxima and one minimum on the latitudinal profile of the electron density calculated from the IRI model was employed using the program. After automatic selection, each event was checked manually to find crest and trough. The EIA parameters have been calculated for each year from 2001 (2002) to 2005 or from 2005 to 2009. The EIA parameter from the IRI-2016 model are computed during 5 most geomagnetic quiet days of each month and 5 year period (2001-2005 and 2005-2009) then grouped into the three seasons: Equinox (March, April, September and October), June solstice (May-August) and December solstice (November-February). The list of quiet days is taken from the website http://wdc.kugi.kyoto-u.ac.jp/qddays/index.html. EIA data for local times from 10:00 LT to 22:00 LT with $1 \mathrm{hr}$ step is considered for further analysis. Finally, obtained values of the EIA parameters from years 2001(2002) - 2005 or 2005-2009 have been averaged to represent local time variation for individual seasons and for high and low solar activity.

\section{Results and Discussion}

In order to study the effect of solar activity on the IRI-2016 model to predict the EIA parameters the period 2001-2009 has been grouped into two parts: 2001-2005 as a moderate activity period and 2005-2009 as low solar activity period. The five-year periods are further grouped into three seasons: Equinoxes, June solstice and December solstice. Figure 2 shows the comparative analysis between the mean crest intensity (MCI) estimated from the IRI2016 model and those observed from CHAMP (top panel) and GRACE (bottom panel) observation., respectively. Figure shows that the IRI-2016 model underestimates MCI values those from the satellite observations (CHAMP and GRACE) throughout 2001-1009. In addition, the difference in MCI between observations and the IRI-2016 model is larger during moderate solar active period 2001-2005 (max. difference 0.80 units with CHAMP) than that during low solar active period 2005-2009 (max. difference 0.60 units with CHAMP). The seasonal analysis shows that the underestimation in MCI by the IRI-2016 model is most prominent during the December solstice.

The comparative analysis of crest-to-trough ratio (CTR) from satellite observations and the IRI-2016 model is shown in Figure 3. The CTR from the IRI model generally are underestimated by the satellite observations and the underestimation in the IRI model is larger during moderate solar active period 2001-2005 (max. difference 2.4 units) than that during the low solar active period 2005-2009. It seems the differences between IRI model prediction and the satellite observations are similar for CHAMP and GRACE. The CTR variation with IRI-2016 model has similar pattern as the satellite observations except during the December solstice in the time interval 19:00-22:00 LT. The CTR values from the IRI-2016 model are closer to unity during the daytime hour 10:00-18:00 LT and rapidly increases during 19:00-22:00 LT with a maximum at around 22:00 LT. It is interesting to note that CTR values from satellite observations as well as the IRI model show a peak in post-sunset hours with the highest value during the December solstice. If one compare local variation of MCI and CTR it can be noted that after the sunset electron density of EIA trough decreases much deeper than electron density of EIA crest which causes the CTR to attain almost the two times the value of daytime [Xiong et al. 2013]. This is due to the well-known fact of strong vertical drift during the pre-reversal enhancement (PRE). This makes CTR to attain higher value during post-sunset hour than that during the daytime hour. Moreover, the plasma drift due to PRE varies with solar activity and is generally higher during the high solar activity years than that during the low solar activity years [Kumar et al., 2016]. In support of this, a linear correlation between post sunset plasma drift and solar activity was reported in previous works [Vichare and Richmond, 2005; Fejer et al., 2008]. Therefore, higher CTR values in post-sunset hour during high solar activity years could be explained. In contrast to the satellite observations, CTR from the IRI-model in postsunset hours shows higher value during low solar activity years than that during high solar activity years. 


\section{North-South asymmetry of EIA from the IRI model}

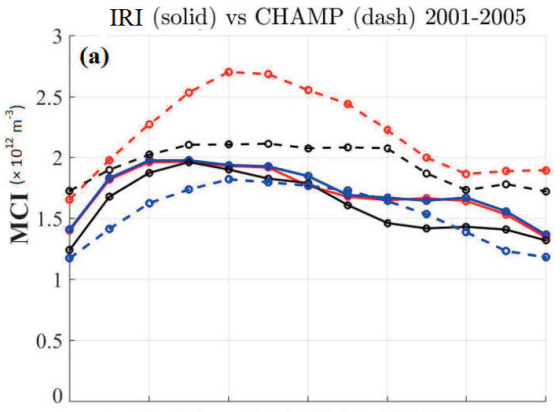

IRI (solid) vs GRACE (dash) 2002-2005

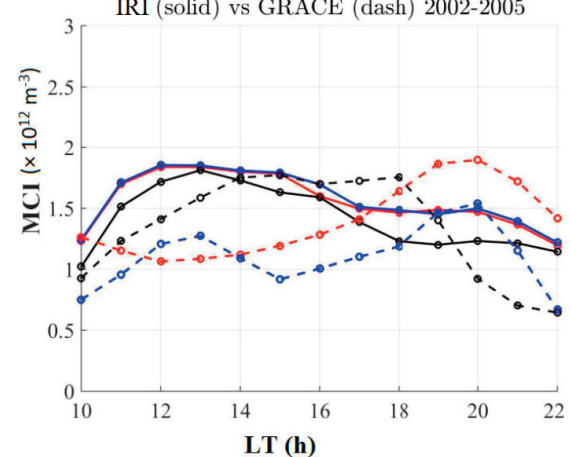

(b)

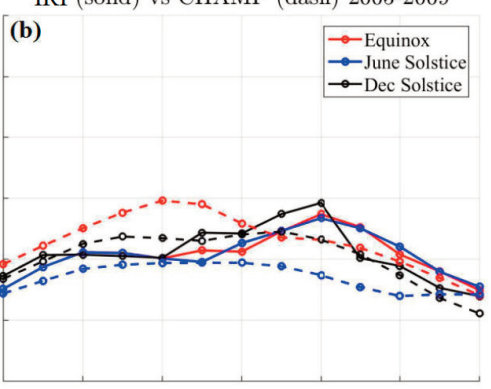

IRI (solid) vs GRACE (dash) 2005-2009

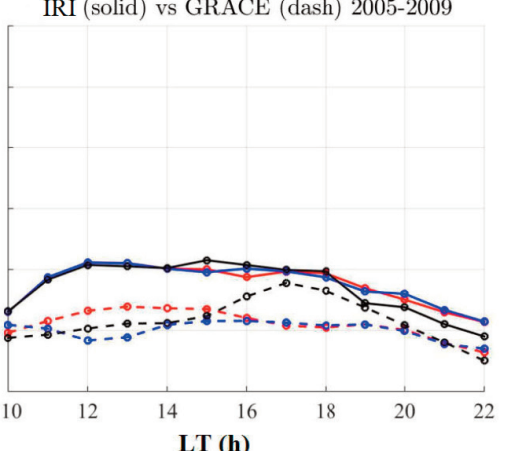

Figure 2. Comparison of mean crest intensity computed during geomagnetic quiet days from the IRI-2016 model with CHAMP (top panels) and GRACE observation during (a) 2001-2005 (b) 2005-2009 (after Xiong et al. [2013]).

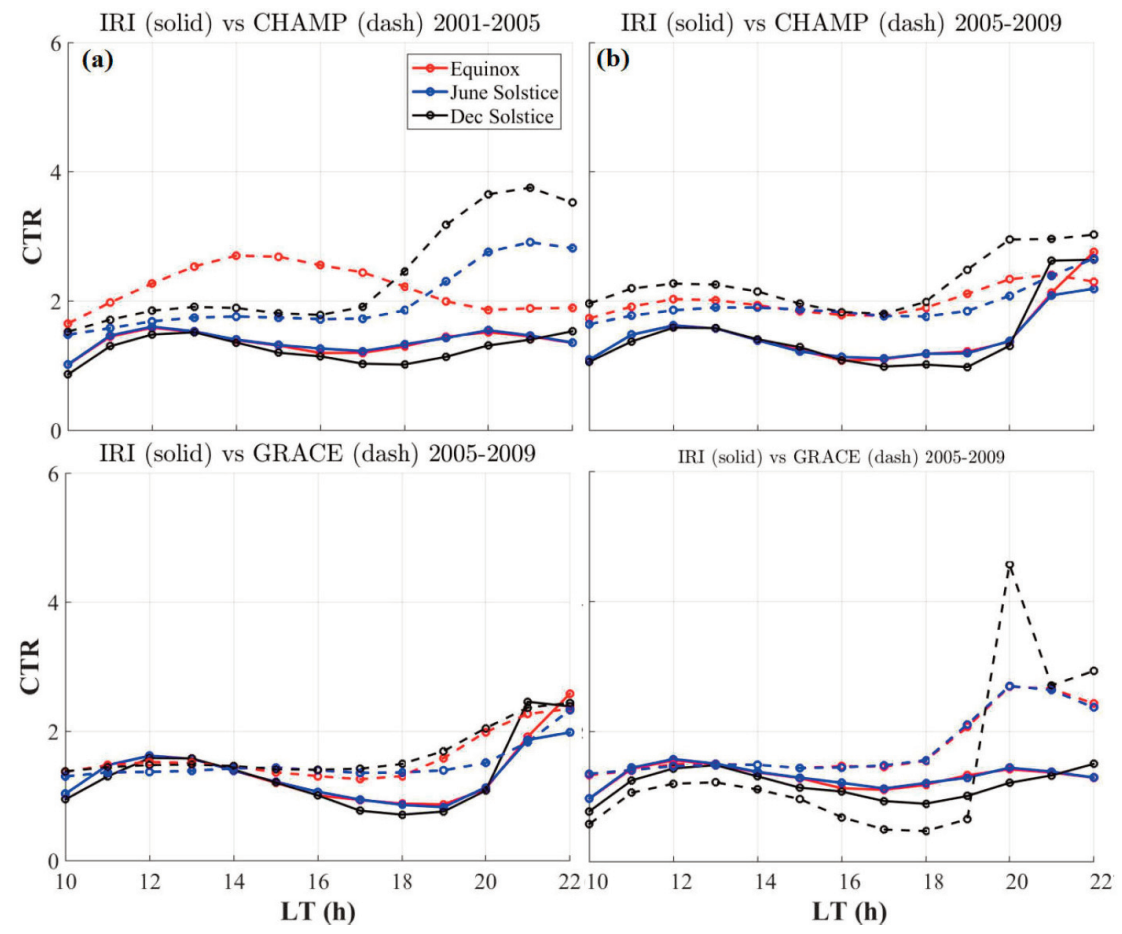

Figure 3. Comparison of crest-to-trough ratio (CTR) computed from the IRI-2016 model with CHAMP (top panels) and GRACE observation during (a) 2001-2005 (b) 2005-2009 (after Xiong et al. [2013]).

Figure 4 shows the comparison of mean crest latitude (MCL) computed from the satellite observations and the IRI2016 model. MCL values from the IRI-2016 model are closer together and underestimate the satellite observations throughout 2001-2009. The difference between the model and satellite observation is found larger during high solar activity period 2001-2005 (max. difference 5.1 unit) than that during the low solar activity period 2005-2009 (max 


\section{Sanjay Kumar}

difference 4.3 unit). The difference between observation and model found maximum during the December solstice. In addition, the IRI-2016 model shows closer agreement with CHAMP observation than the GRACE observation.

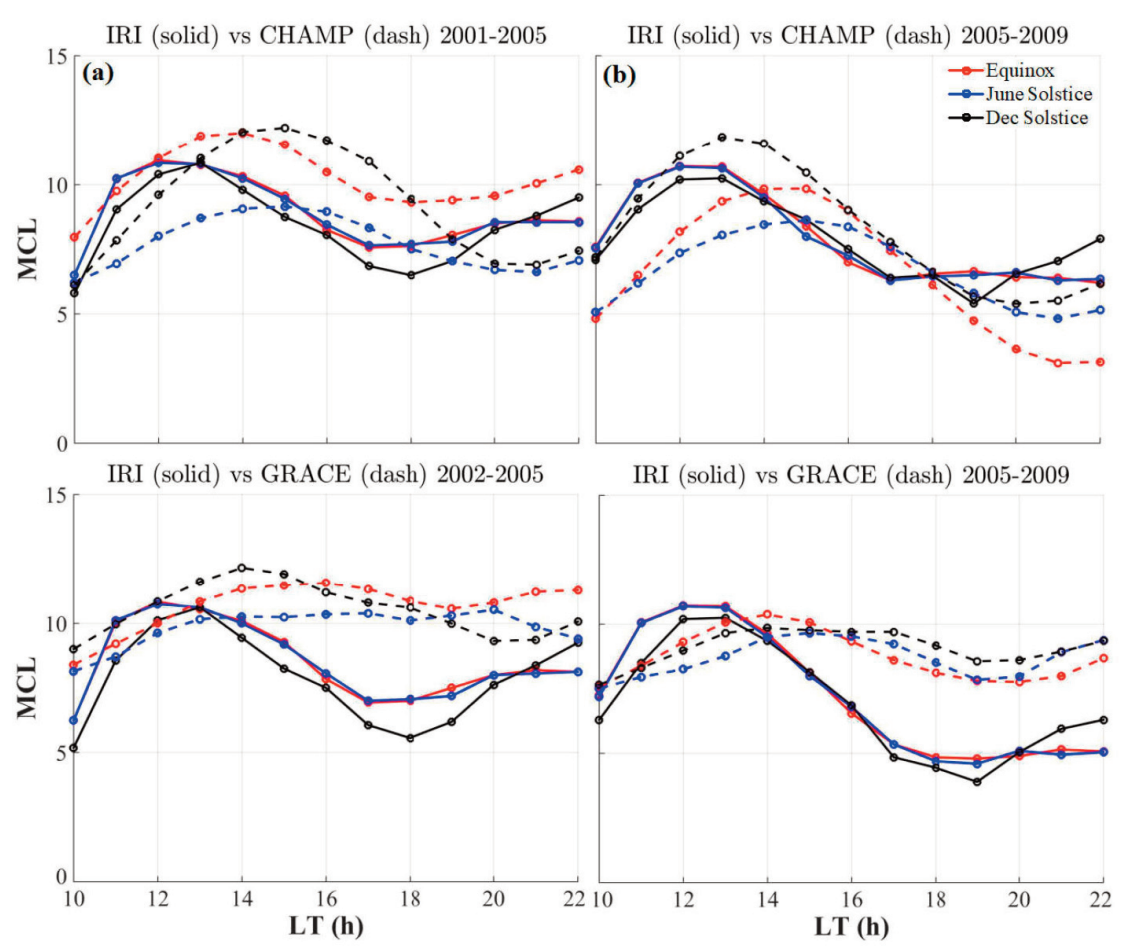

Figure 4. Comparison of mean crest latitude (MCL) computed from the IRI-2016 model with CHAMP (top panels) and GRACE observation during (a) 2001-2005 (b) 2005-2009 (after Xiong et al. [2013]).

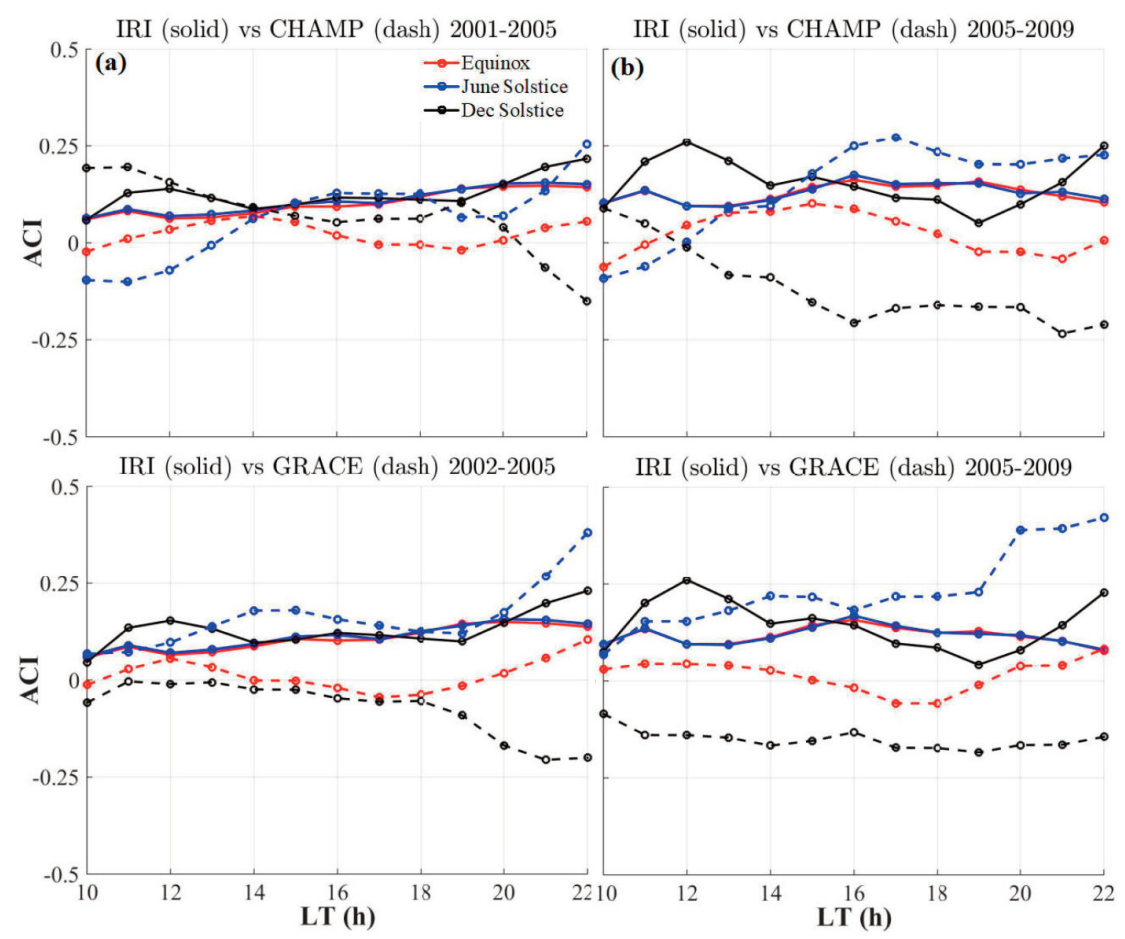

Figure 5. Comparison of asymmetry of crest intensity (ACI) computed from the IRI-2016 model with CHAMP (top panels) and GRACE observation during (a) 2001-2005 (b) 2005-2009 (after Xiong et al. [2013]). 


\section{North-South asymmetry of EIA from the IRI model}

The asymmetry of the crest intensity (ACI) computed from the IRI-2016 model and satellite observation is shown in Figure 5. The ACI values from the IRI-2016 model are closer with satellite observations during moderate solar activity period 2001-2005 than that during the low solar activity period 2005-2009. In addition, the difference between the model and satellite observations are found largest during the December solstice (maximum difference 0.27 unit) and smallest during the June Solstice (difference 0.03 unit). Similarly, asymmetry of the crest latitude (ACL) computed from the IRI-2016 model and a satellite observation is shown in Figure 6. Similar to ACI, the difference of ACL between the IRI-2016 model and satellite observation is larger during low solar activity period 2005-2009 than that during the high solar activity period 2001-2005. In addition, particularly during the June solstice, the nighttime ACL values are significantly larger (difference 7.0 unit) than daytime (difference 2.0 unit). Figure 7 depicts the monthly mean variation of electron density against latitude computed from the IRI-2016 model at an altitude of $400 \mathrm{~km}$ during 2005. From this figure it is clear that the IRI-2016 model failed to predict the NorthSouth asymmetry particularly during the summer solstice months.

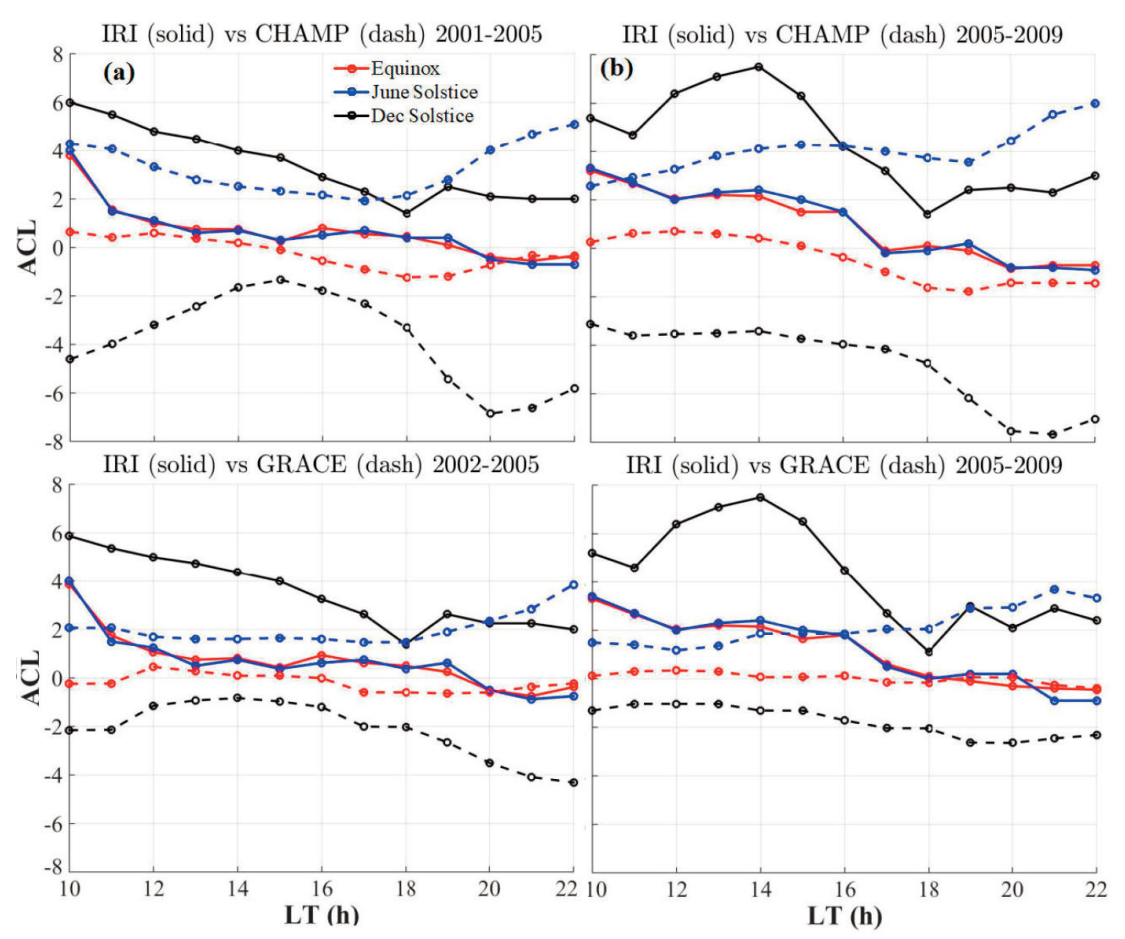

Figure 6. Comparison of asymmetry of crest latitude (ACL) computed from the IRI-2016 model with CHAMP (top panels) and GRACE observation during (a) 2001-2005 (b) 2005-2009. Curves for CHAMP and GRACE are plotted using data from Xiong et al., [2013].

Our results show that the EIA parameters computed from the IRI-2016 model underestimate the satellite observations (CHAMP and GRACE) which are larger during the high solar activity period 2001-2005 than during the low solar activity period 2005-2009 (Figure 4). Similar to our results, the difference between ground-based observations and the IRI model during low and high solar activity years over low-latitude regions of different longitude sectors was reported by previous workers. Their results showed larger discrepancy during high solar activity period as compared to low solar activity period [Kumar et al., 2014b; Venkatesh et al., 2014; Tariku Asmare et al., 2015; Kumar et al., 2015; Kumar, 2016]. Latitudinal migration of EIA crest towards higher latitude with increasing solar activity could be a factor responsible for the larger discrepancy in the IRI model during high solar activity period [Kumar et al., 2014b].

There are basically two major sources for the changes in the electron density distribution over equatorial and EIA regions. One source is the changes in the solar irradiation which further affects the production rate in the ionosphere. Although one of the input parameters of the IRI model is $\mathrm{F}_{10.7}$ solar flux the real solar activity index used 


\section{Sanjay Kumar}

for the IRI-2016 model are: (1) sunspot number, R, (2) global ionospheric index, IG. IG is an ionospheric effective solar index that is based on foF2 measurements from selected ionosondes and a correlation with the CCIR maps. The IRI model uses $F_{10.7}$ solar flux, which is proxy for EUV flux, for representing solar activity influence on ionosphere peak parameters foF2. However, the strength of ionization in the ionosphere is governed by solar EUV irradiance [Kumar, 2016]. Recently, Emmert et al. [2010] have shown the decadal relationship between EUV irradiance and $\mathrm{F}_{10.7}$. They further showed that $\mathrm{F}_{10.7}$ has been changed remarkably (started around 2006) during the recent solar minimum year as compared to previous three decades. The EUV irradiance also decreases more quickly than $\mathrm{F}_{10.7}$ proxy indicating larger difference between $\mathrm{EUV}$ and $\mathrm{F}_{10.7}$ during recent solar minimum.

The second source for electron density variation over equatorial and EIA region is the anomalous variation in the electron density distribution caused by transport-induced effects which include fountain effect (or $\mathbf{E} \times \mathbf{B}$ drift) and trans-equatorial wind induced effect [Appleton, 1946; Kumar et al., 2014a]. At equatorial latitudes, the eastward electric field during daytime in fact is the driving force for the equatorial electrojet (EEJ) and the vertical plasma drift. Furthermore, the strength of the EIA is controlled by the equatorial electrojet (EEJ) strength, which significantly depends on time of a day, day of a year, season, and solar cycle. The changes in EEJ strength can modify the electron density distribution over equatorial and EIA regions [Rama Rao et al., 2006]. Therefore, to include the impacts of the EEJ in the IRI model which controls the EIA and $\mathbf{E} \times \mathbf{B}$ drift is an important job for ionosphere modelers. One possibility to include these impacts in the IRI model is to use the in situ measurements along with ionosonde data in the IRI model for representing true variation in the EIA associated with electric field and neutral wind dynamics. This could be very much useful in progress of the IRI model predictions over equatorial and low latitude regions [Kumar, 2016].

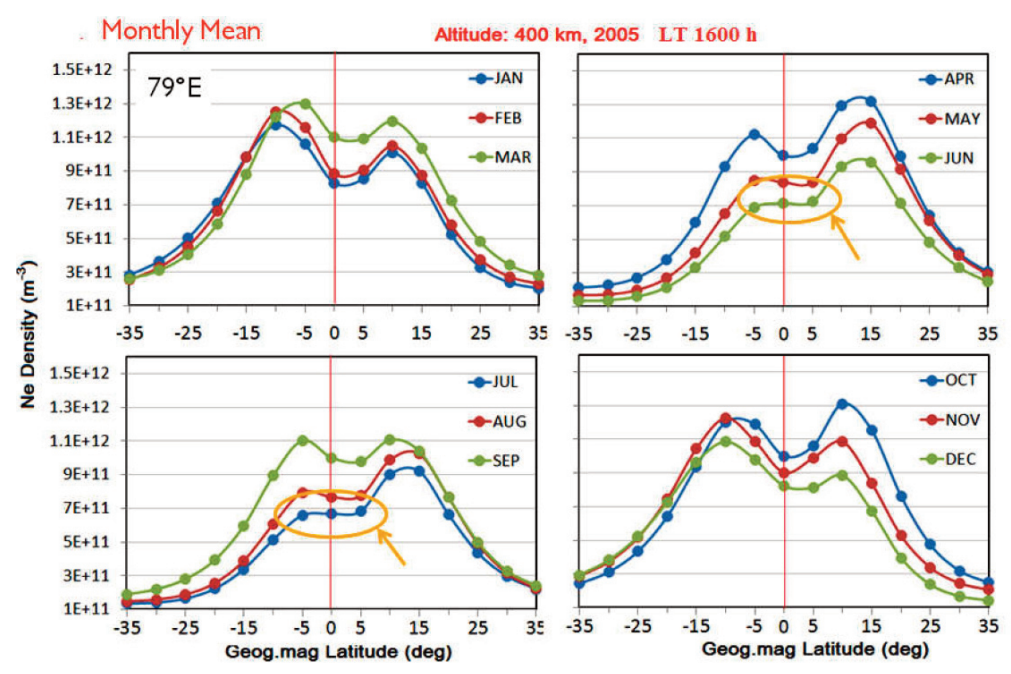

Figure 7. Monthly mean variation electron density estimated during geomagnetic quiet days of each month against latitude computed from the IRI-2016 model at altitude of $400 \mathrm{~km}$, during the high solar active year 2005 .

\section{Summary}

The EIA parameters showing North-South asymmetry has been computed from the IRI-2016 model and compared with those from satellite observations (CHAMP and GRACE) during 2001-2009. The analysed period has been grouped into high solar activity period 2001-2005 and low solar activity period 2005-2009. The findings of this study are listed below:

1. The MCI values from the IRI-2016 model underestimates the MCI values with those from CHAMP and GRACE especially during high solar activity period.

2. The IRI-2016 model has little-to-none noticeable EIA structure during daytime (CTR $\approx 1)$ whereas it goes up to 6 during post-sunset hour especially for low solar activity period. In contrast to the IRI-2016 model, CTR 


\section{North-South asymmetry of EIA from the IRI model}

from satellite is higher during high solar activity period than low solar activity period.

3. The IRI-2016 model estimated MCL values are closer together during all the seasons and underestimates the in-situ measurements CHAMP/GRACE except during the June solstice.

4. The IRI-2016 Model prediction finds positive ACI values which indicate appearance of North crest is favorable. But satellite observations show positive as well as negative ACI.

5. The IRI-2016 generally follows the same latitudinal asymmetry at $400 \mathrm{~km}$ (Same trend of ACL as CHAMP) while it tends to be asymmetric towards the North at $480 \mathrm{~km}$ (GRACE) (Positive ACL). In addition, during the June solstice, the model IRI-2016 shows larger departure from observation after 19:00 UT with larger difference during high solar activity than that during the low solar activity. In addition to this, the IRI-2016 model fails to predict significantly North-South asymmetry (southern crest disappears) for moderate solar activity 2005 . This is an interesting result which needs further investigations.

More data input in the IRI-model particularly in the equatorial region are required so that it could predict correct location of EIA crest which is affected by electric field and neutral wind dynamics.

Acknowledgements. Author is thankful to CSIR, New Delhi for providing financial support under Scientist Pool Scheme (13(9049-A)/2019). The electron density data from the IRI-2016 model have been computed online available at http://irimodel.org/. My sincere thank to Prof. Vladimir Truhlik, Institute of Atmospheric Physics, the Czech Academy of Sciences, Praha, Czech Republic for helping to analyse the IRI data during the IRI-2015 workshop.

\section{References}

Abdu, M.A. (2005). Equatorial ionosphere thermosphere system: electrodynamics and irregularities, Adv. Space Res., $35,771-787$.

Aggarwal, M. (2011). TEC variability near northern EIA crest and comparison with IRI model. Adv. Space Res., 48, 12211231.

Appleton, E.V. (1946). Two anomalies in the ionosphere, Nature, 157, 691.

Balan, N., G. J. Bailey (1995). Equatorial plasma fountain and its effects: possibility of an additional layer, J. Geophys. Res., 100, 21421-21432.

Balan, N., M. Yamamoto, J. Y. Liu, Y. Otsuak, H. Liu, and H. Lühr (2011). New aspects of thermospheric and ionospheric storms revealed by CHAMP, J. Geophys. Res., 116, A07305.

Balan, N., T. Maruyama, A. K. Patra, and V. L. and Narayanan (2018), A minimum in the latitude variation of spread-F at March equinox, Prog. Earth Planet. Sci., 5, 27.

Emmert, J. T., J. L. Lean, and J. M. Picone, J. M. (2010), Record low thermospheric density during the 2008 minimum, Geophys. Res. Lett., 37, L12102, doi:10.1029/2010GL043671.

Fejer, B. G., J. W. Jensen, and S.-Y. Su (2008). Quiet time equatorial F region vertical plasma drift model derived from ROCSAT-1 observations, J. Geophys. Res., 113, A05304, doi:10.1029/2007JA012801.

Heelis, R.A. (2004). Electrodynamics in the low and middle latitude ionosphere:a tutorial, J. Atmos. Sol. Terr. Phys., 66, 825-838.

Huang, Y.N., K. Cheng, and S.W. Chen (1989). On the equatorial anomaly of the ionospheric total electron content near the northern anomaly crest region, J. Geophys. Res. 94, 13515.

Kumar, S., and A. K. Singh (2009). Variation of ionospheric total electron content in Indian low latitude region of equatorial ionization anomaly (EIA), J. Adv. Space Res., 43, 1555-1562.

Kumar, S., S. Priyadarshi, S.Gopi Krishna, and A. K. Singh (2012). GPS-TEC variations during low solar activity period (2007-2009) at Indian low latitude stations, Astrophys. Space Sci., 339, 165-178.

Kumar, S., A.K. Singh, and J. Lee (2014a). Equatorial Ionospheric Anomaly (EIA) and comparison with IRI model during descending phase of solar activity (2005-2009), J. Adv. Space Res., 53, 724-733.

Kumar, S., E. L. Tan, S. G. Razul, C. M. S See, and D. Siingh (2014b). Validation of IRI-2016 model with GPS based ground observation over low-latitude Singapore station, Earth Planet \& Space, 66, 17.

Kumar, S., E. L. Tan, and D. S. Murti (2015). Impacts of solar activity on performance of the IRI-2016 model predictions from low to mid latitudes, Earth Planet \& Space, 67, 42. 


\section{Sanjay Kumar}

Kumar, S. (2016). Performance of IRI-2016 model during a deep solar minimum and a maximum year over global equatorial regions, J. Geophys. Res. Space Physics, 121, 5664-5674.

Sanjay Kumar, W. Chen, Z. Liu, and S. Ji (2016). Effects of solar and geomagnetic activity on the occurrence of equatorial plasma bubbles over Hong Kong, J. Geophys. Res. Space Physics, 121, 9164-9178, doi:10.1002/2016JA022873.

Mannucci, A. J., B. T. Tsurutani, B. A. Iijima, A. Komjathy, A. Saito, W. D. Gonzalez, F. L. Guarnieri, J. U. Kozyra, and R. Skoug (2005). Dayside global ionospheric response to the major interplanetary events of October 29-30, 2003 "Halloween Storms", Geophys. Res. Lett., 32, L12S02.

Martyn, D.F. (1955). Theory of height and ionization density changes at the maximum of a Chapman-like region, taking account of ion production, decay, diffusion and total drift, In: Proceedings Cambridge Conference. Physical Society, London, pp. 254-259.

Mitra, S.K. (1946). Geomagnetic control region F2 of the ionosphere, Nature 158, 668-669.

Moffett, R.J., W. B. Hanson (1965). Effect of ionization transport on the equatorial F region, Nature 206, 705-706.

Raghavarao, R., M. Nageswararao, J. H. Sastri, G. D. Vyas, M. Sriramarao (1988). Role of equatorial ionization anomaly in the initiation of equatorial spread F, J. Geophys. Res. 93, 5959-5964.

Rama Rao, P. V. S., S. Gopi Krishna, K. Niranjan, and D. S. V.V. D. Prasad (2006), Temporal and spatial variations in TEC using simultaneous measurements from the Indian GPS network of receivers during the low solar activity period of 2004-2005, Ann. Geophys., 24, 3279-3292, doi:10.5194/angeo-24-3279-2006.

Rastogi, R.G., J. A. Klobuchar (1990). Ionospheric electron content within the equatorial F2-layer anomaly belt, J. Geophys. Res., 95, 19045-19052.

Rishbeth, H., A. J. Lyon, and M. Peart (1963). Diffusion in the equatorial F layer, J. Geophys. Res., 68, 2559-2569.

Rush, C., M. Fox, D. Bilitza, K. Davies, L. Mcnamara, F. Stewart, and M. Pokempner (1989). Ionospheric mapping - an update of foF2 coefficients, Telecomm J., 56, 179-182.

Stolle, C., C. Manoj, H. Lühr, S. Maus, P. Alken, (2008). Estimating the daytime equatorial ionization anomaly strength from electric filed proxies, J. Gephys. Res., 113, A09310.

Tariku, Y. A. (2015). TEC prediction performance of IRI-2016 model during a very low and a high solar activity phase over equatorial regions, Uganda, J. Geophys. Res. Space Physics, 120, doi:10.1002/2015JA021203.

Venkatesh, K., P. R. Fagundes, G. K.Seemala, R. de Jesus, A. J. de Abreu, and V. G. Pillat (2014). On the performance of the IRI-2016and NeQuick2 models during the increasing phase of the unusual 24th solar cycle in the Brazilian equatorial and low-latitude sectors, J. Geophys. Res. Space Physics, 119, 5087-5105, doi:10.1002/2014JA019960.

Vichare, G., and A. D. Richmond (2005). Simulation studies of the longitudinal variation of evening vertical ionospheric drifts at the magnetic equator during equinox, J. Geophys. Res., 110, A05304, doi:10.1029/2004JA010720.

Vila, P. (1971). New Dynamic Aspects of Inter-tropical $F_{2}$ Ionization, Radio Sci., 6, 945-956, doi:10.1029/RS006i011p00945.

Walker, G.O. (1981). Longitudinal structure of the equatorial ionization anomaly: a review, J. Atmos. Sol-Terr. Phys., 43, 763-774.

Xiong, C., H. Lühr, and S. Y. Ma (2013). The magnitude and inter-hemispheric asymmetry of equatorial ionization anomaly- based on CHAMP and GRACE observations, J. Atmos. Sol-Terr. Phys., (105-106), 160-169.

Xiong, C., Y.-L. Zhou, H. Lühr, H., and S.-Y. Ma (2016). Diurnal evolution of the F region electron density local time gradient at low and middle latitudes resolved by the Swarm constellation, J. Geophys. Res. Space Physics, 121, 9075-9089.

"CORRESPONDING AUTHOR: Sanjay Kumar, 